\section{How systems biology of cancer signaling pathways can inform optimized combination chemotherapy design}

\author{
Jack A. Tuszynski, 1,2 \\ Edward A. Rietman 3 \\ 1DIMEAS, Politecnico di Torino, Torino, \\ Italy; 2Department of Physics, \\ University of Alberta, Edmonton, \\ Canada; 3Department of Computer \\ Science, University of Massachusetts, \\ Amherst, USA
}

\begin{abstract}
This talk presents an overview of the systems biology of cancer applied to specific protein-protein interaction signaling pathways. We argue that network analysis augmented with thermodynamics measures such as Gibbs free energy can lead to optimized design of drug combinations for cancer chemotherapy.
\end{abstract}

\section{Introduction}

The 5-year survival for patients after diagnosis/treatment is strongly dependent on tumor type. For example, prostate cancer patients have a greater than $99 \%$ chance of survival past 5 -years since diagnosis, while pancreatic patients have less than $6 \%$ chance of survival past 5-years. Since each cancer type has its own molecular signaling network, we investigated how "topological signatures" of these networks can inform us about the 5-year survival. We hypothesize that such signatures provide clues for selecting new therapeutic targets. ${ }^{1}$ Using the KEGG Cancer Pathway database we computed several network statistics and found a reasonably high correlation $\left(\mathrm{R}^{2}=0.7\right)$ between degree-entropy $\mathrm{S}$ and 5year survival based on the SEER database. This suggests that cancers that have a more complex molecular pathway network are likely to be more refractory than those with less complex molecular pathway. We also found potential new targets by computing the betweenness centrality, which is a statistical metric of the importance of a node in a molecular network. We have also investigated algebraic and topological indices for network complexity for Protein-Protein Interaction (PPI) networks of various human cancers and found evidence that greater network complexity is associated with lower five-year survival probabilities. Moreover, in this analysis we have found several protein families (e.g. PIK, ITG, AKT, HSP) that represent repeated motives in many of the cancer pathways. Our results can aide in the identification of promising protein targets for anti-cancer drugs.

\section{Materials and Methods}

We used the human protein-protein interaction network ${ }^{2}$ from BioGrid, which contains 9561 nodes and 43,086 edges (http:// thebiogrid.org). The entire human PPI was uploaded into Cytoscape. The list of genes obtained from TCGA (full-length expression set contained 17,814 genes) for a specific cancer was selected using the Cytoscape functions, the inverse selection of Cytoscape function applied, and the nodes and their edges were removed. The resulting network, which included only those genes found in both Biogrid and TCGA, consisted of 7951 nodes and 36,509 edges. This Cytoscape network was then downloaded as an adjacency list for processing with the use of a custom Python code with appropriate NetworkX functions. We used two databases for epidemiological patient survival data: The Surveillance Epidemiology and End Results (SEER) National Cancer Institute database, which contains detailed statistical information about the 5-year survival rates of patients with various types of cancer, and the National Brain Tumor Society database.

Data for several selected cancers from The Cancer Genome Atlas (TCGA) hosted by the National Institute of Health (http://cancergnome.nih.gov) were collected. Specifically, we collected a set of data that used the Agilent platform G4502A and was pre-collapsed on gene symbols. This dataset comprised quantitative information for a total of 11 cancers, namely: KIRC (kidney renal clear cell); KIRP (kidney
Correspondence: Jack A. Tuszynski, DIMEAS, Politecnico di Torino, Torino, Italy; Department of Physics, University of Alberta, Edmonton, Canada.

E-mail: jacek.tuszynski@polito.it

Key words: Systems biology; protein-protein interactions; network analysis; cancer signaling networks; Gibbs free energy; protein expression

Topic: In silico models.

Acknowledgments: This research was partially funded by NSERC (Canada) and the Allard Foundation.

Disclosures: The authors declare no conflict of interest.

Conference presentation: This paper was presented at the Third Centro 3R Annual Meeting - L'era delle 3R: modelli in silico, in vitro e in vivo per promuovere la ricerca traslazionale 30 September - 1 October 2021, Evento online organizzato dal Politecnico di Torino.

Received for publication: 9 July 2021.

Accepted for publication: 7 September 2021.

This work is licensed under a Creative Commons Attribution NonCommercial 4.0 License (CC BY-NC 4.0).

CCopyright: the Author(s), 2021

Licensee PAGEPress, Italy

Biomedical Science and Engineering 2021; 4(s1):143 doi:10.4081/bse.2021.143

renal papillary cell); LGG (low-grade glioma); GBM (glioblastoma multiforme), COAD (colon adenocarcinoma); BRCA (breast invasive carcinoma); LUAD (lung adenocarcinoma); LUSC (lung squamous cell); UCEC (uterine corpus endometrial); OV (ovarian serous cystadenocarcinoma) and READ (rectum adenocarcinoma).

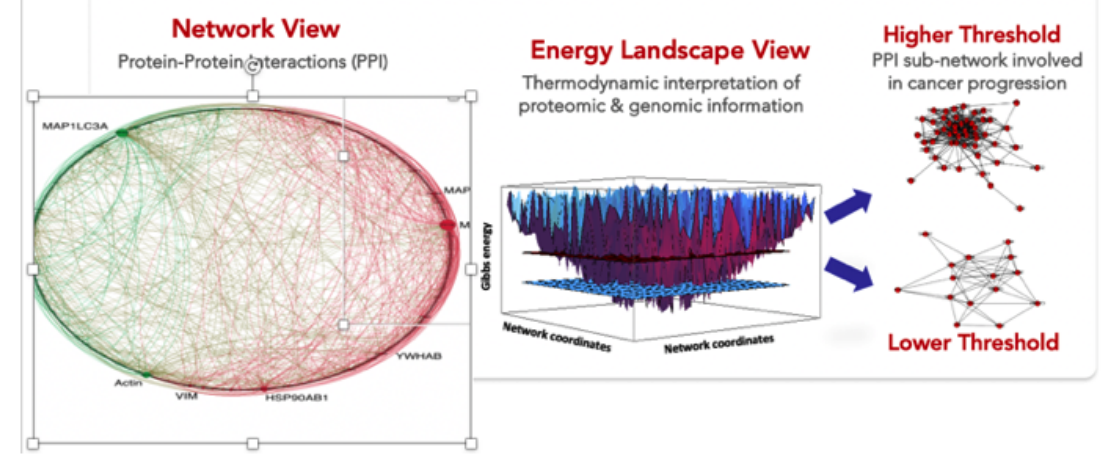

Figure 1. A schematic illustration of the methodology used in this research where PPI is shown with over-expressed and under-expressed proteins and their interactions followed by the generation of a Gibbs free energy landscape and completed by truncation of the network using appropriate thresholds that reveal the key players and their connections. 


\section{Results}

The dynamics of cell activities including cell division, growth and apoptosis are coordinated and controlled by protein-protein interaction which are cancer-type and indeed patient-specific. A complete set of PPIs for a given cancer generates a network with nodes representing proteins and edges their interactions. The state-of-the-art database of these PPI networks is BioGRID. Since the proteome has not been fully mapped from open-reading frames to genes and proteins, calculations of these networks' mathematically described properties such as network entropy or the Gibbs free energy should be taken as estimates reflecting the present state of knowledge about these networks. Here, we report the outcomes of merging two types of data, transcriptome and PPI networks, to compute the energetic state of each particular cancer. We show a correlation between the Gibbs free energy and 5-year patient survival for different cancers using the methodology illustrated in Figure 1.

\section{Discussion and Conclusions}

As information about cancer-related genomic alterations emerge and more and more data become available, we can now endeavor to establish the relationships between PPI network complexity and cancer progression on an individual patient basis. We provide Gibbs free energy, a thermodynamic measure encompassing both network complexity and protein concentration (transcriptome), and show that this thermodynamic measure can be correlated with cancer survival. This allows us to potentially differentiate between normal and cancer cells using thermodynamic measures. Moreover, this method can be used to design a chemotherapy regimen based on a combination of drugs that minimize the thermodynamic measures of the corresponding PPI network's complexity ${ }^{3,4}$ via an optimization approach. The method has been validated in vitro ${ }^{5}$ and efforts are underway for clinical implementation of this methodology to improve therapeutic outcomes.

\section{References}

1. Breitkreutz D, Hlatky L, Rietman E, Tuszynski JA. Molecular signaling network complexity is correlated with cancer patient survivability. Proc Natl Acad Sci USA 2012;109:9209-12.

2. Rietman EA, Platig J, Tuszynski JA, Lakka Klement G. Thermodynamic measures of cancer: Gibbs free energy and entropy of protein-protein interactions. J Biol Phys 2016;42:339-50.

3. Benzekry S, Tuszynski JA, Rietman EA, et al. Design principles for cancer therapy guided by changes in complexity of protein-protein interaction networks. Biol Direct 2015;10:32.

4. Carels N, Tilli T, Tuszynski JA. A computational strategy to select optimized protein targets for drug development toward the control of cancer diseases. PLoS One 2015;10:e115054.

5. Tilli TM, Carels N, Tuszynski JA, Pasdar M. Validation of a network-based strategy for the optimization of combinatorial target selection in breast cancer therapy: siRNA knockdown of network targets in MDA-MB-231 cells as an in vitro model for inhibition of tumor development. Oncotarget 2016;7: 63189-203. 